\title{
Dynamic Analysis For Power Amplifier Electronic Equipment
}

\author{
Famao $\mathrm{Wu}^{\mathrm{a}}{ }^{*}$, Long Jiang , Jian Wang and Weibing Zhu \\ School of Mechanical Engineering ,XiHua University.Chengdu,Sichuan,610039,China \\ awufamao@126.com
}

Keywords: power amplifier electronic equipment; vibration isolation system; modal analysis; random vibration analysis; dynamics analysis

\begin{abstract}
Power amplifier electronic equipment is an important component of aviation weapon system, and random vibration is a main factor result in structural failure. In order to fully understand dynamic characteristics of power amplifier electronic equipment, according to modal analysis theory, the theory of random vibration analysis, constraint method and the equivalent treatment of shock absorber and so on, for dynamic simulation analysis of power amplifier electronic equipment. Each natural frequency and principal mode of vibration is obtained through the modal analysis $t$, these results provide the basis for subsequent fixture design requirements. Through random vibration analysis to understand the dynamic characteristics of power amplifier electronic equipment, provide a reference for subsequent shock absorber selection.
\end{abstract}

\section{Introduction}

With the rapid development of military electronic technology, military electronic devices are moving toward the diversification, high performance and high reliability; miniaturization, lightweight of the product structure rapid development. In the 1950s, aerospace electronic devices in the aircraft of the total cost is less than $10 \%$ percent, but in the 90 's, with the improvement of performance of the aircraft, airborne electronic equipment in the aircraft as a proportion of the total cost has risen to $30 \% \sim 40 \%$, some airborne early warning and electronic warfare aircraft has reached 50\%[1]. Electronic device during transport and in the course of work, will to varying degrees under the influence of various factors, such as weather, temperature, vibration, shock, and its internal structure fatigue etc. Due to vibration caused by electronic equipment structure and damage to components on the printed circuit board is most prominent. According to statistics, electronic equipment damage due to vibration factors accounted for $27 \%$ [2-3]. Electronic equipment machine comprehensive performance is good or bad will directly affect the overall operational performance of the flight. In order to achieve the high reliability of electronic devices and the design of high performance requirements, need dynamic simulation analysis was carried out on the electronic equipment, through the simulation analysis to understand the dynamic characteristics, so as to predict it in the course of the work, then carries on the corresponding damping buffer design, to improve the quality of products, improve product performance.

\section{Power amplifier electronic equipment structure characteristics and model simplification}

\subsection{Structural characteristics of power amplifier electronic equipment}

Amplifier electronic equipment is mainly composed of shock absorber, installation frame, function module, rack, heat sinks, fans, etc. and the function module consists of aluminum plate, perimeter aluminum frame, printed-circuit board (PCB), connectors, power supply, etc. Electronic equipment chassis through the Guide pins of installation on the back side plates and locking device installed in the installation frame; Shock absorber by screws are connected to the installation frame. Power amplifier of electronic equipment vibration isolation system consists of installation frame and shock absorber, Simplified CAD model of power amplifier electronic devices is shown in figure 1 . 


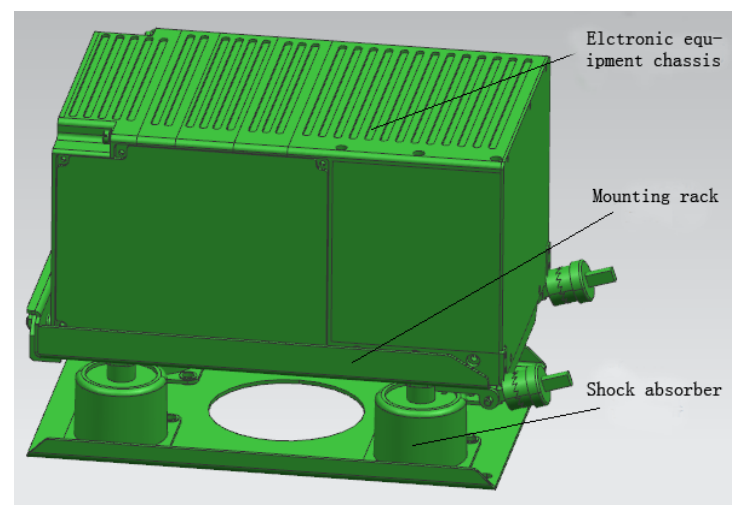

Fig.1. Power amplifiers electronic equipment

\subsection{Model simplification of power amplifier electronic equipment}

Model simplification is a very important stage of finite element analysis, quality of model simplification will be directly related to the mesh quality. Mesh quality directly affects the efficiency of finite element analysis. Electronic equipment model is more complex assembly structure, including non-bearing parts, bearing parts and decorative parts, and some tiny features, these tiny characteristics are hardly the focus of concern. If directly on the model meshing, not only can make the grid size, and easy to form the deformed mesh, can lead to the time of solution increase and even solve failure. So model simplification is necessary before mesh generation, and model simplification process should pay attention to the following two aspects: (1) Model simplification should guarantee the accuracy of the model size enough, should as far as possible to ensure that the equipment structure stiffness does not change. (2) There are many small features in the model component, such as holes, chamfers, bosses etc. which have little effect on results should be deleted.

\section{Mesh and constrained load}

\subsection{Finite element model}

Mesh quality on the precision of the simulation results and the convergence of the solution process is important, and unit number excessive can make computer slow, unit number too little can cause low accuracy of simulation results. Power amplifier electronic equipment overall dimension: $286 \mathrm{~mm} \times$ $212 \mathrm{~mm} \times 198 \mathrm{~mm}$. According to different size of model use different mesh size: $2 \mathrm{~mm}, 3 \mathrm{~mm}$, mesh generation adopt tetrahedral mesh division, after division of finite element model the total number of cells for 336,984, total number of nodes to 637,165, the finite element model is shown in Figure 2.

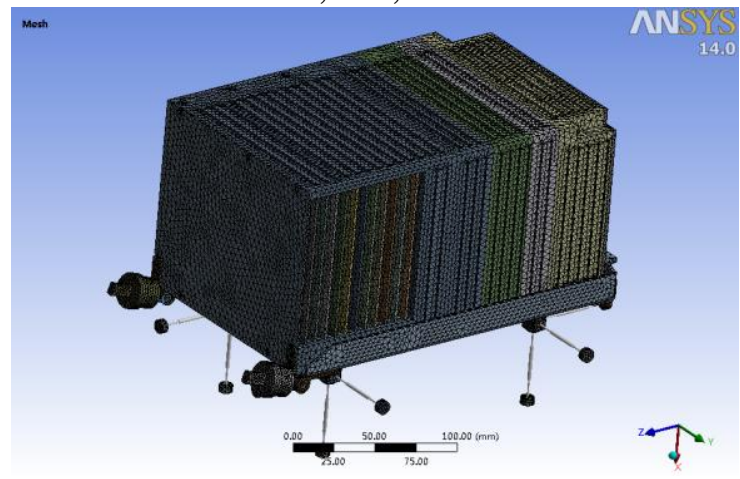

Fig.2. Finite element model

\subsection{The equivalent treatment and constraints imposed the shock absorber}

The amplifier electronic devices is using metal helical spring shock absorber, and the shock absorber internal structure is complex, it is difficult to through solid modeling for simulation analysis. Using 
spring simulate the shock absorber in software, only consider the spring stiffness coefficient $k$ and damping coefficient ${ }^{c}$.In practical work, metal coil spring shock absorber stiffness coefficient is larger than the other two main directions. In the simulation analysis, the other stiffness in both directions and generally the main direction stiffness coefficient of $80 \%$ [4]. Through theoretical calculations be stiffness and damping coefficients as shown in table 1.

\section{Table 1}

Vibration isolator characteristic parameter

\begin{tabular}{ccc}
\hline Name & $k(\mathrm{~N} / \mathrm{m})$ & $c(\mathrm{~N} \cdot \mathrm{s} / \mathrm{m})$ \\
\hline The main direction & 22700 & 300 \\
Lateral direction & 18160 & 240 \\
\hline
\end{tabular}

Power electronic equipment is connected to aircraft through the shock absorbers, during actual installation process, shock absorber is through the four mounting holes connect to pedestal.

\section{Modal analysis of power amplifier electronic equipment}

\subsection{Theory of the modal analysis}

Modal analysis is based on vibration theory for the study of dynamic characteristics of the equipment, identify the frequency characteristics of the equipment. Modal analysis is based on three basic assumptions [5]: linear assumption, observability assumption and time invariance assumption [6]. $\mathrm{N}$ degrees of freedom matrix linear differential equation:

$$
[M]\{\ddot{x}\}+[C]\{\dot{x}\}+[K]\{x\}=\{F\}
$$

Where $[M]$ is the mass matrix, $[C]$ is the damping matrix, $[K]$ is the stiffness matrix; $\{\ddot{x}\}$ is the acceleration response vector, $\{\dot{x}\}$ is the speed response vector, $\{x\}$ is the displacement vector, $\{F\}$ is the vibration force vector. To type (1) both sides transformation, available:

$$
\left(s^{2} M+s C+K\right) X(s)=F(s)
$$

That $s=j \omega$, Fourier transform, modal coordinates can be obtained the equation of motion:

$$
\left[K-M w^{2}+j C w\right] X(w)=F(w)
$$

response at any point is the linear combination of modal responses, namely:

$$
X_{l}(\omega)=\varphi_{l 1} q_{1}(\omega)+\varphi_{l 2} q_{2}(\omega)+\cdots+\varphi_{l r} q_{r}(\omega)+\cdots+\varphi_{l N} q_{N}(\omega)
$$

where $q_{r}(\omega)$ is the $\mathrm{r}$-order modal coordinates, $\varphi_{l r}$ is the $\mathrm{r}$-order mode shape factor, then, $\mathrm{r}$-order mode shape factor column vector of $\mathrm{N}$ measured points can be expressed as:

$$
\phi_{r}=\left[\varphi_{r 1}, \varphi_{r 2}, \cdots, \varphi_{r N}\right]^{\mathrm{T}}
$$

modal matrix : $\Phi=\left[\phi_{1}, \phi_{2}, \cdots, \phi_{N}\right]$, then, model coordinate vector is:

$$
Q=\left[q_{1}(\omega), q_{2}(\omega), \cdots, q_{N}(\omega)\right]^{\mathrm{T}}
$$

the modal coordinates: $\mathrm{X}(\omega)=\Phi Q$, free damped vibration system equation can be expressed as: 


$$
\left(K-\omega^{2} M+j C \omega\right) \Phi Q=F(\omega)
$$

The equation of motion can be decoupled, and it is a basic equations of dynamics, and each equation represents a single degree of freedom systems, they are independent of each other, and can be obtained by solving the equations of the natural frequency of the structure characteristic parameters.

\subsection{Results of modal analysis}

In $0 \sim 2000 \mathrm{~Hz}$ within the scope of the electronic device there is more than one resonance frequency, resonant frequency of some components is not of concern. Here concerned only with the first 10 frequencies, table 2 for power amplifier model first 10 modal frequency parameters of electronic equipment. If power electronic devices as a rigid body, the device would be equivalent to an elemental, it has a direction along the axis direction and around the axis, and each campaign has its own natural frequency. Figure 3 for 3-order mode shape of the amplifier electronics.

Table 2

Modal frequency and modal shapes of modal analysis

\begin{tabular}{ccc}
\hline Number & Frequency $/ \mathrm{Hz}$ & Vibration mode \\
\hline 1 & 11.99 & The whole machine Translational vibration along the $\mathrm{Y}$ direction \\
2 & 15.20 & The whole machine Translational vibration along the $\mathrm{Z}$ direction \\
3 & 23.18 & The whole machine Translational vibration along the $\mathrm{X}$ direction \\
4 & 26.72 & The whole machine rotation vibration around the $\mathrm{X}$ axis \\
5 & 36.45 & The whole machine rotation vibration around the $\mathrm{Y}$ axis \\
6 & 37.71 & The whole machine rotation vibration around the $\mathrm{Z}$ axis \\
7 & 265.2 & Mounting bracket(1 order modal) bending deformation on the $\mathrm{X}$ direction \\
8 & 437.9 & Mounting bracket(2 order modal) bending deformation on the $\mathrm{X}$ direction \\
9 & 488.4 & Mounting bracket(3 order modal) bending deformation on the $\mathrm{X}$ direction \\
10 & 588.7 & The side plate bending deformation on the $\mathrm{Z}$ direction \\
\hline
\end{tabular}

Resulting cloud pictures of modal analysis as follows:

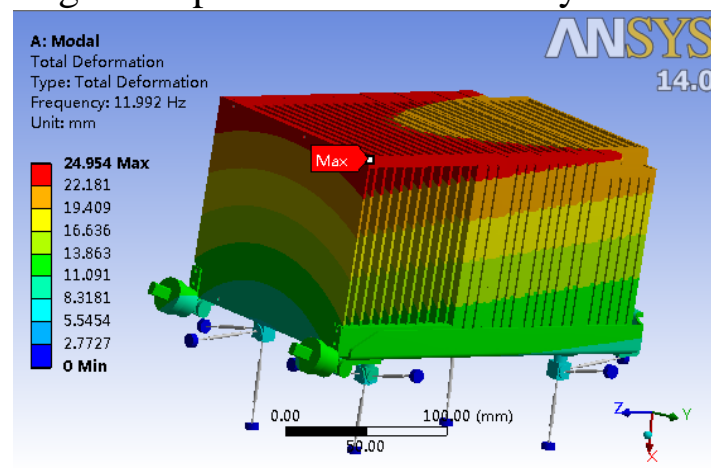

(a) modal 1

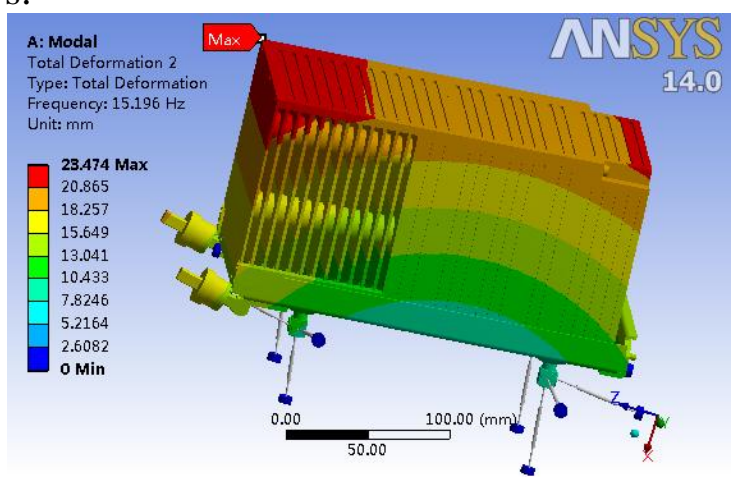

(b) modal 2

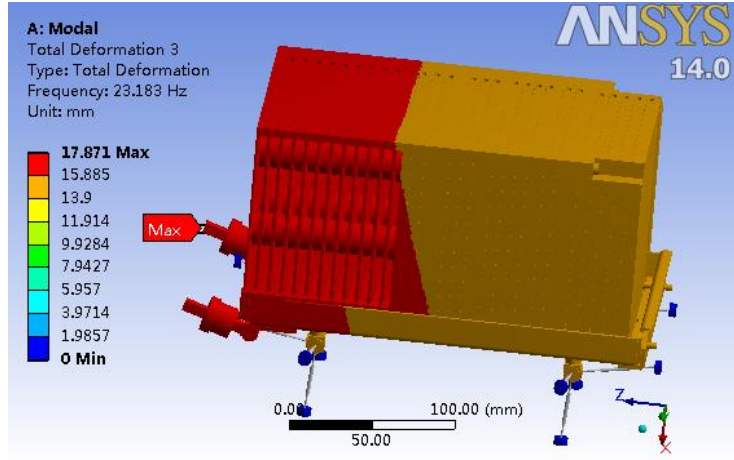

(c) modal 3

Fig.3. First 3 modal shapes of the modal analysis 


\section{Random vibration analysis}

\subsection{The principle of random vibration}

Random vibration analysis also known as the power spectral density analysis, is a spectrum analysis based on probability statistics technology, is a statistical sample that time into a power spectral density function (PSD). Random vibration analysis of power spectral density (PSD) recorded excitation and response of root mean square values relations with the frequency, so the PSD is a power spectral density value-frequency curves, that is, load time history. Power spectral density is a frequency domain description of random variable autocorrelation function, can reflect the frequency of random loads. $x(t)$ is this function, then autocorrelation functions can be expressed as:

$$
R(\tau)=\lim _{T \rightarrow+\infty} \frac{1}{T} \int_{-T / 2}^{T / 2} x(t) x(t+\tau) d t
$$

when $\tau=0$, Autocorrelation function takes a maximum value equal to the mean square value of random load: $R(0)=E\left(x^{2}(t)\right)$ 。

Due to ${ }^{R(\tau)}$ two sufficient conditions of Fourier transform, so autocorrelation function and power spectrum density function form the forward and inverse Fourier transform, that is, namely:

$$
\left\{\begin{array}{l}
\mathrm{S}(f)=\int_{-\infty}^{\infty} R(\tau) \mathrm{e}^{-\mathrm{i} 2 \pi f \tau} d \tau \\
R(\tau)=\int_{-\infty}^{\infty} S(f) \mathrm{e}^{\mathrm{i} 2 \pi f \tau} d f
\end{array}\right.
$$

where $f$ is the frequency, $\mathrm{S}(f)$ is the power spectral density function of $x(t)$, power spectral density curves describing the power spectral density $\mathrm{S}(f)$ relationship with frequency $f$.

\subsection{Results of random vibration analysis}

Using software ANSYS Workbench simulation on random vibration analysis of the power electronic equipment, acceleration power spectral density (PSD G Acceleration) inertial load is imposed on spring mounting base fixed of finite element models, separately on the voyage direction, the lateral and vertical (in Figure 2 correspond to Y,X,Z) three directions. Obtained response results of power amplifier electronic equipment by random vibration simulation analysis, figure 4 is the whole machine acceleration response cloud pictures.

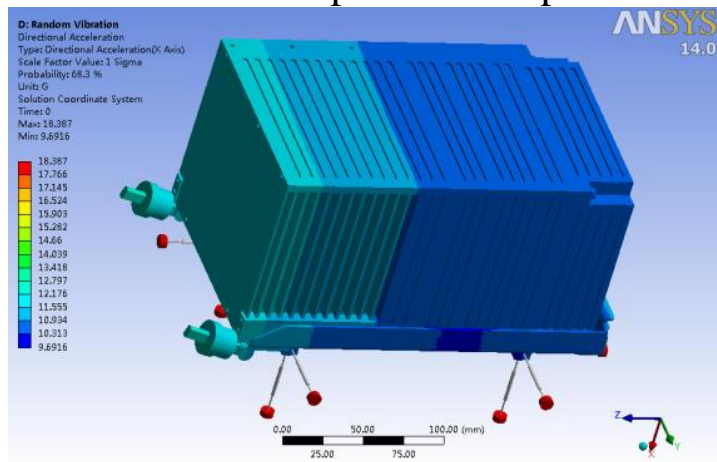

(a) $\mathrm{X}$ direction acceleration response images

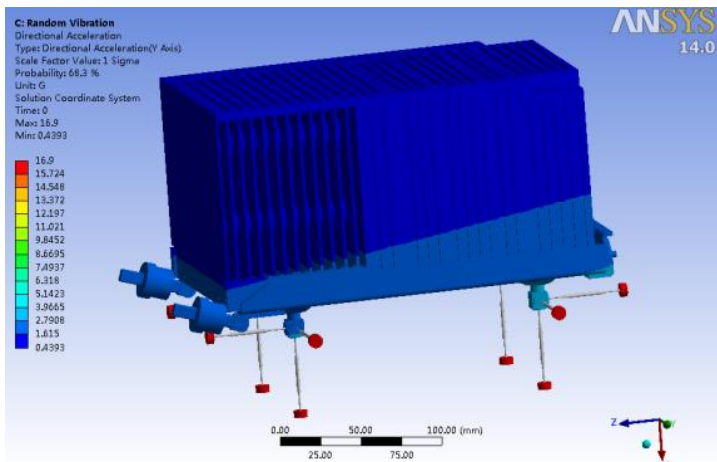

(b) Y direction acceleration response images 


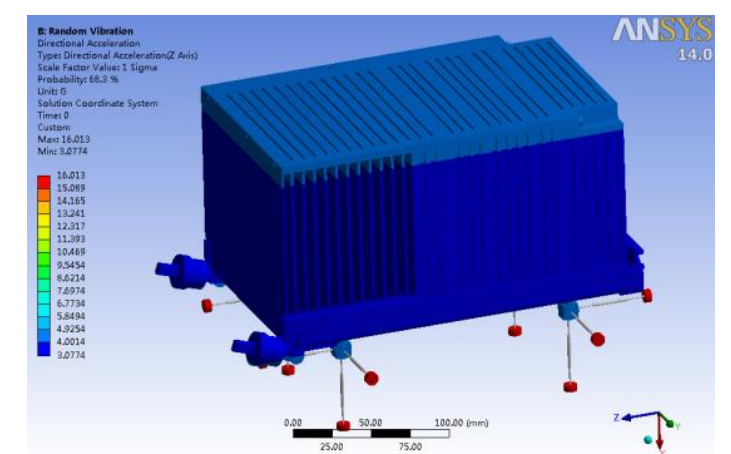

(c) $\mathrm{Z}$ direction acceleration response images

Fig. 4. Acceleration response cloud pictures of the whole machine

\section{Summary}

(1) From the power amplifier electronic equipment model the first 10 order modal frequency can be seen that the first 6 order modal frequency is very small and is between $11 \sim 38 \mathrm{~Hz}$, the former 6 frequencies are very close, to prevent vibration system coupling between phenomenon, natural frequency of the internal components begin in 7th modal frequency. Namely, the 1-order natural frequency of internal components is $265.2 \mathrm{~Hz}$, its mode is on the mounting rack X-direction bending deformation. Based on modal analysis of power electronic equipment, obtained the inherent frequency and vibration mode, provide the design basis for the vibration test fixture design.

(2) From power amplifier electronic equipment whole machine random vibration simulation analysis cloud images can be found that the $\mathrm{X}$ direction to the overall magnitude was greater than other two directions, and the vibration base incentive level is also the biggest, the problem in the process of actual test also mainly appear in the direction. So the installation frame optimization design and vibration isolation system test mainly carry out in X direction.

(3) From the $\mathrm{X}$ direction clouds pictures of acceleration response can also be seen that on the top of the equipment the front and rear the acceleration response difference is very big, the front end (front locking device corresponding to the surface) vibration magnitude is larger. When carrying out vibration tests, sensors can be installed at the front and rear of equipment. These can also provide a reference for type selection of shock absorber.

\section{Acknowledgement}

This work was supported by Xihua University graduate student Innovation Fund (No.ycjj2015076) and the Key Project of Sichuan Education Department (No.15ZA0126).

\section{References}

[1] Huoman, Deng Zhongwei. Development trend of foreign military aircraft avionics systems [J]. Avionics, 2004.12,35 (4): 5-10.

[2] Li Chaoxu. Vibration resistance design of electronic equipment [J]. Electronic mechanical engineering, 2002.3,18 (1): 51-55.

[3] Risto Hienonen,Matti Karjalainen,Raija Lankinen.Verification of the Thermal Design of Electronic Equipment[M].Espoo:Technical Research Centre of Finland,1997:13-86.

[4] Zhang Yinghui, Liu Huihang, Wang Decheng. Spring brochure [M]. Beijing: China machine press, 1997.6.

[5] Xu Wenben, Jiao Qunying. Mechanical vibration and modal analysis [M]. Beijing: China machine press, 1998:80-88.

[6] Zhang Lingmi. Development of dynamic test technology seminar series [J]. Vibration and shock, 1998.8,3 (17): 83-85. 